\section{Correlation of 3DCRT and Open Field Treatment Techniques in Advanced Head and Neck Cancer Patients Treated by Chemoradiation}

\author{
Anjali Sachan ${ }^{1 \oplus}$, Seema Gupta ${ }^{1 *}$, Navin Singh ${ }^{2}$
}

\begin{abstract}
Background: Multimodality treatment is required for the management of head and neck cancer. Functional impairment and toxicities associated with surgery and radiation accentuate the need to develop innovative therapeutic strategies in the management of these patients to improve survival and reduce toxicity. In this study, we have compared treatment effects in patients of advanced head and neck squamous cell cancer treated by open field and 3DCRT treatment planning techniques.
\end{abstract}

Objective: This study aims to evaluate open field and 3DCRT treatment planning techniques in advanced head and neck squamous cell cancer patients managed by chemoradiation in the scenario of limited resources.

Material and Methods: In this analytical study, 40 histologically proven unresectable advanced squamous cell carcinoma patients of oropharynx and larynx were allocated in two groups to receive 70 Gy in 35 fractions in 7 weeks with concurrent cisplatinum $35 \mathrm{mg} / \mathrm{m}^{2}$ weekly either with open-field technique or three dimensional conformal radiotherapy (3DCRT) by ElektaSynergy linear accelerator. Target volume coverage and dose received by organ at risk (OARs) were compared. Clinical outcome in terms of response and toxicities is also evaluated in this study.

Results: Plans with best possible coverage of the target volume were obtained. No significant difference was found in the dose received by the spinal cord; however, it was possible to prevent higher dose to brain stem with 3DCRTin node negative patients of oropharynx cancer and larynx cancer. Skin toxicities were significantly lower in 3DCRT arm.

Conclusion: In low resource settings with increased burden of locally advanced disease, both open-field and 3DCRT treatment techniques are comparable in terms of target coverage, OARs preservation, toxicity and treatment response.

Citation: Sachan A, Gupta S, Singh N Correlation of 3DCRT and Open Field Treatment Techniques in Advanced Head and Neck Cancer Patients Treated by Chemoradiation. J Biomed Phys Eng. 2022;12(1):83-90. doi: 10.31661/jbpe.v0i0.2007-1132.

\section{Keywords}

Head and Neck Cancer; Radiotherapy, Conformal; Radiation Dosage; Radiation Effects; Dose Response

\section{Introduction}

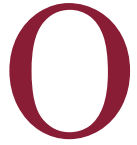
rgan-preservation protocols using combined chemoradiation have become the acceptable definitive treatment of advanced malignancy of head and neck [1]. Radiotherapy planning and treatment delivery in head and neck malignancy have now progressed with the advent of computer optimized radiation techniques. The con-
${ }^{1} \mathrm{MD}$, Department of $\mathrm{Ra}$ diotherapy, King George's Medical University, Lucknow, Uttar Pradesh, India

${ }^{2} \mathrm{PhD}$, Department of Radiotherapy, King George's Medical University, Lucknow, Uttar Pradesh, India

*Corresponding author: Seema Gupta

Department of Radiotherapy, King George's Medical University, Lucknow Uttar Pradesh, India

E-mail: seemaguptart@ gmail.com

Received: 1 July 2020 
formal radiotherapy has enabled to deliver more accurate curative dose to the tumour and can prevent critical structures by limiting dose to organs at risk [2-5].

Radiation in head and neck malignancy is effortful and requires vigilant monitoring because large fields are frequently used to treat primary disease and regional drainage lymph node in neck. In established radiotherapy treatment planning, tumor should receive the prescribed dose and critical structures around should receive feasibly less dose. This pattern of radiation delivery is possible by using conformal radiotherapy specifically by intensitymodulated radiotherapy (IMRT); however, I MRT technique of radiation delivery is very sensitive to any change, occurring during treatment period [6].

Likelihood of developing toxicity depends on dose per fraction, volume irradiated, site of radiation, concurrent chemotherapy used and patient's performance status.Xerostomia is uppermost late toxicity which patients receive after the head and neck radiation. It affects swallowing, speech and oral hygiene, which ultimately deteriorate patient's wellbeing.

Acute radiation toxicities in the head and neck cancer are mucositis, dysphagia and trismus. They occur frequently and result in remarkable clinical signs and symptoms in those who are grievously affected by the disease in head neck cancer.

Studies have shown better sparing of paroti$\mathrm{dgl}$ and the use of IMRT in contrast to nonIMRT radiation techniques, with substantial reduction in xerostomia [6]. Nevertheless, there are alterations during the treatment period with IMRT as a result of actual delivered dose that may not correspond to planned dose resulting in increased radiation toxicities. These variations, needing review in the treatment planning make changes in body weight, tumour reduction and regression in parotid gland volume. Therefore, taking these variations into account adaptive radiotherapy may have important implication [7]. However, the prevalent current paradigm of conventional radiotherapy is predominantly persisting because of technology limitations, increased patient numbers and limited resources available with patients to afford the expenses.

Therefore, our aim in this study was to evaluate and compare the treatment effect in patients of advanced malignancy of head and neck treated by 3DCRT and conventional radiotherapy planning.

\section{Material and Methods}

This was analytical study wherein 40 patients with stage II-IV of American Joint Committee on Cancer's (AJCC cancer staging manual of $8^{\text {th }}$ edition) head and neck squamous cell cancer, registered at department of radiotherapy, King George's Medical University, Lucknow were prospectively enrolled. Eligible patients had newly diagnosed biopsy proven head and neck squamous cell cancer; on clinical examination, they had gross primary and neck nodes, which was also anticipated in contrast enhanced computed tomography. All these patients received concomitant cisplatin $35 \mathrm{mg} / \mathrm{m}^{2}$ weekly and were randomized in two groups as a study groupand Bas a control group to receive radiation by 3DCRTandconventional planning technique, respectively. Study informed consent was taken from all the patients.

The study was approved by the ethical review board $\left(90^{\text {th }}\right.$ ECM II B-Thesis/P11).

\section{Randomization}

Patients were allocated in 1:1 ratio to either open field (control arm) or 3DCRT (study arm) by simple random sampling method [Table 1].

Treatment planning, delivery and follow-up

3DCRT was planned and delivered in 2 phases, 46 Gy in 23 fractions for 5 weeks in phase 1 and 24 Gy in 12 fractions for 2 weeks in phase 2 to receive a total tumour dose of 70 Gy in 35 fractions over 7-weeks. Each patient 
Table 1: Distribution of cases in two arms

\begin{tabular}{ccc} 
& Frequency & Percent \\
\hline 3DCRT & 21 & 52.5 \\
\hline Open & 19 & 47.5 \\
\hline Total & 40 & 100.0
\end{tabular}

3DCRT: Three-Dimensional Conformal Radiotherapy

underwent a planning kilo voltage computerized tomography scan (kVCT-scan) (Brilliance CT, Phillips Medical Systems Nederland B.V.) of the head-and-neck region with a 3-mm slice thickness. The patients were scanned in the supine position and immobilized on a flat table top with a customized five fixation point's thermoplastic facemask and a head-and-neck immobilization board (AIO Board). Planning kVCT images were transferred to Xio treatment planning system, and contours for the target volumes and normal organs were drawn.

Planning CT with intravenous contrast was obtained with $3 \mathrm{~mm}$ slice thickness from vertex to carina. Subsequently, target volumes and adjacent normal critical structures were manually contoured on axial slice of planning CT scan. Gross tumour volume (GTV) was delineated as mass displayed in enhanced CT images, and it included primary tumour (GTV$\mathrm{P})$ and enlarged regional neck nodes (GTV-N).

The clinical target volume (CTV) was defined as the GTV plus a margin of probable microscopic spread. High-risk nodal regions were included. For treatment planning, the planning target volume (PTV) circumscribed CTV with a 5-mm margin. Beam arrangements consisted of five/ six co-planar beams to deliver 70 Gy in 35 fractions over 7 weeks in two phases to PTV by Elekta Synergy linear accelerator. Inbuilt universal wedges were applied as and when required beams were weighted accordingly to reduce hot spots. The dose constraints were set on the organs at risk accordingly. Open field in conventional planning technique was planned using conventional simulator (simulix evolution, Elekta) and delivered total dose of 70 Gy in 35 fractions over 7 weeks in 2 phases, 46 Gy in 23 fractions for 5 weeks in phase 1 and 24 Gy in 12 fractions for 2 weeks in phase 2 .

All patients received weekly chemotherapy with cisplatin $\left(50 \mathrm{mg} / \mathrm{m}^{2}\right)$ concurrent with radiotherapy. Patients were examined weekly during therapy to assess treatment-related toxicities. All patients were on a diet plan as advised by a dietician. Patients were provided symptomatic and supportive care as and when required during and following the treatment. Patients were followed up 4 weeks from completion of treatment for assessment of response and subsequently kept on regular follow-up.

\section{Statistical Analysis}

The data was analyzed by SPSS software version 22. Age and sex of individuals are represented as mean with standard deviation. Various topographical locations of tumor sites and etiological factors are presented in frequencies. Comparison of both the groups, with respect to PTV coverage area and dose distribution to organs at risk around tumor area was done by independent t-test. Association with clinical outcomes in terms of loco-regional response; toxicity was done by a chi-square analysis. For all the tests, p-values less than 0.05 were considered as statistically significant.

\section{Results}

The present study assesses the treatment response and treatment related toxicities in locally advanced head and neck cancer patients treated by 3DCRT and conventional radiotherapy planning technique.

Total 40 patients were recruited. All these patients received concomitant cisplatin 35 $\mathrm{mg} / \mathrm{m}^{2}$ weekly and were randomized in two groups, including A as a study group and B as a control group to receive radiation either by 3DCRT or conventional planning technique, respectively. Radiotherapy was performed with total dose of 70 Gy in 35 fractions over 
7 -weeks in 2 phases in both the groups [Table $2]$.

\section{Patient's population}

A total number of 40 patients in locally advanced stage III and stage IV carcinoma base of tongue, tonsil, soft palate, larynx, supraglottic larynx and subglottic larynx were enrolled and allocated randomly by simple random sampling method in equal numbers in open-field radiotherapy and 3DCRT treatment groups [Table 2]. After completion of treatment, these patients were followed up by three months. There is a need to assess the longterm treatment effect in the two techniques of radiotherapy treatment delivery system in the future.

No significant difference was found between the treatment group A (3DCRT) and B group (conventional radiotherapy) with reference to age, sex, prognostic factors, tumour size, tumour grade, tumour differentiation, and tumour stage.

\section{Patient's characteristics \\ Demographic Profile}

The demographic characteristics of head and neck squamous cell cancer (HNSCC) patients at presentation (enrolment) is summarized in Table 3.

Mean and median age were $51.93 \pm 10.31$ and 52 years. Hence, 53 were taken as the cutoff of age.

Tumour details

Total of 2 sites were included in the study. Oropharynx was the most commonly involved site $(65 \%)$ and base of tongue was the most commonly involved sub site $(45 \%)$. Maximum cases were of T3 stage (65\%). Distribution of cases according to node involvement is given in Table 2. The most of patients had stage 4A disease followed by stage 3, accounting together for $77.5 \%$ of tumours. $72.5 \%$ of the patients had moderately differentiated tumours followed by $22.5 \%$ of well differentiated and $5 \%$ of poorly differentiated tumours
Table 2: Tumor details of head and neck squamous cell cancer patients

\begin{tabular}{ccc} 
Variable & Frequency & Percent \\
\hline Site: & & \\
\hline LARYNX & 14 & 35.0 \\
\hline OROPHARYNX & 26 & 65.0 \\
\hline Total & 40 & 100.0 \\
\hline
\end{tabular}

Sub site:

\begin{tabular}{ccc}
\hline BASE OF TONGUE & 18 & 45.0 \\
\hline SOFT PALATE & 3 & 7.5 \\
\hline $\begin{array}{c}\text { SUPRAGLOTTIC LAR- } \\
\text { YNX }\end{array}$ & 14 & 35.0 \\
\hline TONSIL & 4 & 10.0 \\
\hline VALLECULA & 1 & 2.5 \\
\hline Total & 40 & 100.0 \\
\hline Grade: & & \\
\hline M/D & 29 & 72.5 \\
\hline P/D & 2 & 5.0 \\
\hline W/D & 9 & 22.5 \\
\hline Total & 40 & 100.0
\end{tabular}

Tumor Stage:

\begin{tabular}{ccc}
\hline T1 & 1 & 2.5 \\
\hline T2 & 4 & 10.0 \\
\hline T3 & 26 & 65.0 \\
\hline T4A & 7 & 17.5 \\
\hline T4B & 2 & 5.0 \\
\hline Total & 40 & 100.0 \\
\hline
\end{tabular}

Nodal Class:

\begin{tabular}{ccc}
\hline N0 & 8 & 20.0 \\
\hline N1 & 8 & 20.0 \\
\hline N2 & 1 & 2.5 \\
\hline N2A & 6 & 15.0 \\
\hline N2B & 2 & 5.0 \\
\hline N2C & 8 & 20.0 \\
\hline N3B & 7 & 17.5 \\
\hline Total & 40 & 100.0 \\
\hline Nodal Stage & 15 & 37.5 \\
\hline IVA & 16 & 40.0 \\
\hline IVB & 9 & 22.5 \\
\hline Total & 40 & 100.0
\end{tabular}

IVA, IVB; Nodal tumor stage 
Comparative Study Open Field and 3DCRT

[Table 2].

Treatment effect

The effect of treatment of the patients in both the arms in terms of tumor response, toxicity, target dose coverage, dose to critical structures is summarized in Tables 3 and 4.

PTV Coverage

The volume of PTV covered by $95 \%$ of the isodose curve was $97.44 \%$ in the 3DCRT conformal group while it was $98.34 \%$ in the con-

Table 3: Distribution of patients by radiation induced dermatitis and mucositis and response

\begin{tabular}{|c|c|c|c|c|}
\hline Variables & $\begin{array}{c}\text { Open Technique } \\
\text { (Frequency) }\end{array}$ & $\begin{array}{c}\text { 3DCRT Technique } \\
\text { (Frequency) }\end{array}$ & $X^{2}(\mathrm{df})$ & p-value \\
\hline \multicolumn{5}{|c|}{ Age } \\
\hline$<52$ & 10 & 10 & \multirow{2}{*}{$0.1 .00(1)$} & \multirow{2}{*}{0.752} \\
\hline$\geq 52$ & 9 & 11 & & \\
\hline \multicolumn{5}{|c|}{ Sex } \\
\hline Male & 19 & 2 & \multirow{2}{*}{$0.358(1)$} & \multirow{2}{*}{0.550} \\
\hline Female & 16 & 3 & & \\
\hline
\end{tabular}

Skin Toxicity

\begin{tabular}{|c|c|c|c|c|}
\hline Grade1 & 1 & 12 & \multirow{3}{*}{$13.201(2)$} & \multirow{3}{*}{0.001} \\
\hline Grade 2 & 16 & 9 & & \\
\hline Grade 3 & 2 & 0 & & \\
\hline \multicolumn{5}{|c|}{ Mucosa Toxicity } \\
\hline Grade1 & 1 & 1 & \multirow{3}{*}{$1.591(2)$} & \multirow{3}{*}{0.451} \\
\hline Grade 2 & 9 & 14 & & \\
\hline Grade 3 & 9 & 6 & & \\
\hline \multicolumn{5}{|c|}{ Tumor response } \\
\hline Grade1 & 9 & 12 & \multirow{3}{*}{$0.530(2)$} & \multirow{3}{*}{0.767} \\
\hline Grade 2 & 3 & 2 & & \\
\hline Grade 3 & - & - & & \\
\hline
\end{tabular}

3DCRT: Three-Dimensional Conformal Radiotherapy

Table 4: Mean target coverage and doses to organ at risks in two arms.

\begin{tabular}{ccccc} 
& \multicolumn{4}{c}{ Radiotherapy Technique } \\
\cline { 2 - 5 } & $\begin{array}{c}\text { Open Technique } \\
(\mathbf{N}=19) \text { Mean } \pm \text { SD }\end{array}$ & $\begin{array}{c}\text { 3DCRT (N=21) } \\
\text { Mean } \pm \text { SD }\end{array}$ & Df & P-value \\
\hline PTV Coverage & $98.34 \% \pm 0.014$ & $97.44 \% \pm 0.013$ & 38 & 0.822 \\
\hline Spinal Cord & $44.00 \pm 10.07$ & $44.71 \pm 1.11$ & 38 & 0.097 \\
\hline Brain Stem & $42.88 \pm 10.41$ & $41.30 \pm 7.96$ & 38 & 0.294 \\
\hline Left Parotid & $51.98 \pm 13.66$ & $47.95 \pm 5.70$ & 38 & 0.202 \\
\hline Right Parotid & $51.75 \pm 13.83$ & $46.74 \pm 10.20$ & 38 & 0.866
\end{tabular}

SD: Standard Deviation, 3DCRT: Three-Dimensional Conformal Radiotherapy, Df: Degree of freedom, PTV: Planning Target Volume 
ventional group, which was clinically insignificant by independent t-test [Table 4].

Preservation of Parotid Glands

Parotid glands could not be effectively preserved in patients of either group [Table 4]. As bilateral parallel and opposed lateral fields were applied in both the treatment techniques to treat the clinically enlarged lymph nodes as well as subclinical group of lymph nodes on both 1 sides.

In an attempt to prophylactically irradiate the neck nodes bilaterally due to possibility of subclinical lymph node involvement in locally advanced cases of oropharynx and larynx carcinoma, parotid gland could not be saved substantially even with the conformal 3DCRT radiotherapy technique.

Moreover, the parotid glands were found to be in close proximity with the enlarged level $1 \mathrm{~B}$ and level 2 lymph nodes. Furthermore, part of parotid gland was found to be included in PTV final and in some cases even in the CTV nodal or CTV primary.

Dose received by Spinal Cord

The maximum dose obtained by spinal cord collectively after completion of both phases of treatment was $44.00 \pm 10.07$ in the conventional group, and in the 3D CRT group, it was $44.71 \pm 1.11$ with a p-value of 0.097 , which was not clinically significant [Table 4].

\section{Brain Stem Dose}

The maximum dose received by the brain stem in the 3DCRT arm was $41.30 \pm 7.96$ while in the open-field arm was $42.88 \pm 10.41$ with a p-value of 0.294 that was not clinically significant [Table 4].

Dose to brain stem in patients of carcinoma larynx and oropharynx with no clinically evident lymphadenopathy was lower in 3DCRT technique than that in open-field technique. However, in patients with clinically evident level 2 or 5 lymphnodes, not much reduction in the dose to brain stem was found with either of the two techniques.

Toxicity

Nearly, all patients developed treatment related skin toxicities, out of which grade 1 skin toxicities, were more in 3DCRT arm while grade 2 skin toxicities were found to be more in conventional arm [Table 3]. Few patients developed toxicity which required discontinuation or interruption of radiation. Such patients were managed symptomatically with intravenous hydration of 2 to 2.5 liters of normal saline and dextrose. Supportive treatment in the form of intravenous amino acids was also infused. Patients were given proper dietary advice, keeping in view of their total caloric requirement and difficulties, faced by patients in eating due to the local disease, trismus or radiation mucositis. Patients were also supported with parenteral nutrition and nasogastric tube or Ryles tube insertion was done in some patients as and when required.

The frequency and grading of toxicity viz dehydration, mucositis, anemia and pain during radiation were comparable in both the groups.

The rate of mucositis was found to be equal in both the technique ( $p$ value $>0.05$ ) while rate of skin toxicity was statistically higher in open field or conventional arm with a $p$ - value of 0.001 .

\section{Tumor Response}

Complete clinical response of the primary tumor was seen in 9 patients treated with open field while in 12 patients treated with 3DCRT technique. Chi square analysis showed no significant difference in clinical response between two treatment group ( $p$ value 0.767 ) [Table 3].

Partial response was obtained in 7 patients in each arm.

The rate of tumor response does not significantly differ according to tumor site, size, stage, initial tumor dimensions, and type of radiotherapy technique used for treatment.

\section{Discussion}

Chemoradiation is established organ preservation management of locally advanced oropharyngeal and laryngeal carcinoma $[8,9]$. Most of conformal radiotherapy techniques have assisted in delivering immense accurate 
curative radiation doses to the tumor, and by that it restricts dose delivered to organs at risk; thus, treatment related morbidity is reduced substantially. The probability of radiation toxicity depends on dose per fraction, duration of treatment and volume of normal tissue irradiated [10].

Xerostomia is most notable, unmanageable and bothersome late toxicity which is commonly apparent in the patients of head and neck cancer treated by radiation. Radiation of head and neck cancer gives rise to difficulty in speech, swallowing and also increase the risk of dental caries due to reduced saliva flow [11]. Manifestation of xerostomia is determined by the technique of delivering radiation which in sequence will influence total dose received by parotid glands and volume of parotid glands irradiated.

Studies have documented reduced incidence of xerostomia when the doses to parotids were minimized $[12,13]$.

Acute radiation toxicities in head and neck cancer are mucositis, dysphagia, trismus.

Acute toxicities occurs frequently in radiation of head and neck cancer and results in significant clinical manifestations in those who are severely affected by disease.

In this study, we have tried to compare treatment outcome in locally advanced head and neck cancer patients treated by 3DCRT and conventional radiotherapy planning techniques.

It has been found that there was no significant difference in planning target volume coverage of tumour, dose to organ at risk and treatment response in both the arms; however, skin toxicity increased significantly in patients treated by conventional planning technique. Radiation delivery technique and the type of energy use are not the only factors which are responsible in determining the response of a tumour to the treatment given. Apart from tumour stage, there are some other factors, influencing the response of tumour to the radiation treatment viz radiobiological behaviour of the tumour, degree of differentiation, mitotic activity, hypoxia and expression of growth factors [14]. However, 3DCRT technique is relatively easy to be implemented and is cost effective as well [15]. Furthermore, the results obtained from treatment analysis of 3DCRT can be used as a groundwork and may form based on comparing the improvements obtained with IMRT [16].

\section{Conclusion}

Open-field or conventional technique still forms the backbone of treatment delivery in low resource settings with heavy patient burden and proves to be helpful in successfully treating the patients with less expenditure of time and money.

3DCRT conformal technique has been beneficial in early stages, but in locally advanced stages of head and neck cancers, it seems to be equivalent to open field in terms of preservation of organ at risks and treatment response. Nevertheless, normal tissue complications in terms of skin toxicities and mucosal toxicities were less with 3DCRT.

Our study helps to understand that conventional and 3D conformal radiotherapy techniques still are a major and effective tool for managing head and neck cancers in developing regions, facing a major problem of resources, technical and scientific advances, scarcity of manpower, massive disease burden and poor patient compliance.

It is concluded that in centers of resource constraint where IMRT is not available or for patients who cannot use IMRT technique, treatment with 3DCRT technique would be practicable and feasible option considering locoregional response and related toxicity.

\section{Authors' Contribution}

Anjali Sachan collected data, prepared manuscript drafty. Seema Gupta designed the manuscript, collected data, prepared manuscript drafty. Navin Singh collected data and analyzed them. All the authors read, modified, and approved the final version of the manuscript. 


\section{Ethical Approval}

Approved by the ethical review board (90th ECM II B-Thesis/P11).

\section{Informed consent}

Informed consent was taken.

\section{Conflict of Interest}

None

\section{References}

1. Braakhuis BJ, Brakenhoff RH, René Leemans C. Treatment choice for locally advanced head and neck cancers on the basis of risk factors: biological risk factors. Ann Oncol. 2012;23(suppl 10):x173-7. doi: 10.1093/annonc/mds299. PubMed PMID: 22987957.

2. Purdy JA. 3-D conformal radiotherapy: a new era in the irradiation of cancer. 29th Annual San Francisco Cancer Symposium; San Francisco, Calif: Basel, Karger; 1996. p. 1-16.

3. Purdy JA. From new frontiers to new standards of practice: advances in radiotherapyplanning and delivery. Front Radiat Ther Oncol. 2007;40:18-39. doi: 10.1159/000106026.

4. Purdy JA. Advances in the planning and delivery of radiotherapy: new expectations,new standards of care. Front Radiat Ther Oncol. 2011;43:1-28. doi: 10.1159/000322396.

5. Thiagarajan A, Caria N, Schöder H, Iyer NG, Wolden $\mathrm{S}$, Wong RJ, et al. Target volume delineation in oropharyngeal cancer: impact of PET, MRI, and physical examination. Int J Radiat Oncol Biol Phys. 2012;83(1):220-7. doi: 10.1016/j.jijobp.2011.05.060. PubMed PMID: 22035663.

6. Gupta T, Kanna S, Laskar SG, Agarwal J P. Systematic review and meta-analyses of intensity-modulated radiation therapy versus conventional two-dimensional and/or or three-dimensional radiotherapy in curativeintent management of head and neck squamous cell carcinoma. PLoS One. 2018;13(7):e0200137. doi: 10.1371/journal.pone.0200137. PubMed PMID: 29979726. PubMed PMCID: PMC6034843.

7. Morgan HE, Sher DJ. Adaptive radiotherapy for head and neck cancer. Cancers Head Neck. 2020;5:1. doi: 10.1186/s41199-019-0046-z. PubMed PMID: 31938572. PubMed PMCID: PMC6953291.

8. Denis F, Garaud P, Bardet E, Alfonsi M, Sire C, Germain T, et al. Final results of the 94-01 French Head andNeck Oncology and Radiotherapy Group randomized trial comparing radiotherapyalone with concomitant radiochemotherapy in advanced-stage oropharynxcarcinoma. J Clin Oncol. 2004;22(1):69-76. doi: 10.1200/ JC0.2004.08.021. PubMed PMID: 14657228.

9. Wendt TG, Grabenbauer GG, Rödel CM, Thiel HJ, Aydin H, Rohloff R, et al. Simultaneous radiochemotherapyversus radiotherapy alone in advanced head and neck cancer: a randomizedmulticenter study. J Clin Oncol. 1998;16(4):1318-24. doi: 10.1200/ JC0.1998.16.4.1318. PubMed PMID: 9552032.

10. Hölscher T, Bentzen SM, Baumann M. Influence of connective tissue diseases on the expression of radiation side effects: A systematic review. Radiother Oncol. 2006;78(2):123-30. doi: 10.1016/j.radonc.2005.12.013.

11. Wijers OB, Levendag PC, Braaksma MMJ, Boonzaaijer M, Visch LL, Schmitz PIM. Patients with head and neck cancer cured by radiation therapy: A survey of the dry mouth syndrome in long-term survivors. Head Neck. 2002;24(8):737-47. doi: 10.1002/hed.10129. PubMed PMID: 12203798.

12. Nutting CM, Morden JP, Harrington KJ, Urbano TG, Bhide SA, Clark C, et al. Parotid-sparing intensity modulated versus conventional radiotherapy in head and neck cancer (PARSPORT): A phase 3 multicentre randomised controlled trial. Lancet Oncol. 2011;12(2):127-36. doi: 10.1016/S14702045(10)70290-4. PubMed PMID: 21236730. PubMed PMCID: PMC3033533.

13. Deasy JO, Moiseenko V, Marks L, Chao KSC, Nam $\mathrm{J}$, Eisbruchet A. Radiotherapy Dose-Volume Effects on Salivary Gland Function. Int J Radiat Oncol Biol Phys. 2010;76(3):S58-63. doi: 10.1016/j. ijrobp.2009.06.090. PubMed PMID: 20171519. PubMed PMCID: PMC4041494.

14. Joseph B, Kumar RV, Champaka G, Shenoy A, Sabitha $\mathrm{KS}$, Lokesh V, et al. Biological tailoring of adjuvant radiotherapy in head and neck and oral malignancies - The potential role of p53 and elF4E as predictive parameters. Indian J Cancer. 2019;56(4):330-4. doi: 10.4103/ijc.IJC_56_18. PubMed PMID: 31607702.

15. Herrassi MY, Bentayeb F, Malisan MR. Comparative study of four advanced 3d-conformal radiation therapy treatment planning techniques for head and neck cancer. J Med Phys. 2013;38(2):98-105. doi: 10.4103/0971-6203.111331. PubMed PMID: 23776314. PubMed PMCID: PMC3683308.

16. Lee N, Akazawa C, Akazawa P, Quivey JM, Tang C, Verhey $\mathrm{LJ}$, et al. A forward-planned treatment technique using multisegments in the treatment of head-and-neck cancer. Int J Radiat Oncol Biol Phys. 2004;59(2):58494. doi: 10.1016/j.jijrobp.2004.02.005. PubMed PMID: 15145180. 